\title{
Hepatitis $C$ virus prevalence among patients with thalassemia and inherited bleeding disorders in Iran: a systematic review and meta-analysis
}

\begin{abstract}
Background: Hepatitis C Virus (HCV) as the most common causes of post transfusion infections, is a major global health problem due to liver cirrhosis and hepatocarcinoma. $\mathrm{HCV}$ prevalence varies from each region of the world to another and is more prevalent in patients receiving blood transfusions. Hence, Patients with thalassemia and inherited bleeding disorders are at high risk of $\mathrm{HCV}$ infection. This systematic review and metaanalysis study aimed to determine Hepatitis C Virus prevalence among patients with thalassemia and inherited bleeding disorders in Iran.
\end{abstract}

Methods: Comprehensive searches were carried out in databases including PubMed, EMBASE, the Scientific Information Database (SID) of Iran, the World Health Organization Index Medicus for the Eastern Mediterranean Region (IMEMR WHO), Ebsco, Science Citation Index Expanded, Ovid, Google Scholar, Iran Medex and Magiran up to January 2017. The findings were reported following PRISMA guidelines. The pooled proportion rates were calculated using a Metaprop program on Stata version 14.1 for Mac. Also, the confidence intervals of each study were calculated using the exact method.

Results: Forty-seven studies, composed of 12449 patients; 8673 thalassemia and 3776 patients with inherited bleeding disorders (mainly hemophilia), fulfilled our criteria and included in the study. The year of data collected from the studies was between 1998 to 2015 . The pooled HCV prevalence was estimated $28 \%$ generally ( $95 \%$ confidence intervals $[\mathrm{CI}]=22 \%-33 \%$ ) and the $\mathrm{HCV}$ prevalence in patients with thalassemia and inherited bleeding disorders were 19\% ([CI] 95\%=16\%-23\%) and $42 \%$ ([CI] 95\%=33\%-52\%), respectively. There was a considerable heterogeneity between studies.

Conclusion: Our findings indicated the high prevalence of HCV among patients with thalassemia and inherited bleeding disorders with a decreasing trend of past studies in Iran. Moreover, it is significantly higher in patients with inherited bleeding disorders than patients with thalassemia. In comparison with similar studies, the prevalence of $\mathrm{HCV}$ infection in Iran is low.

Keywords: Hepatitis C virus, Thalassemia, Inherited bleeding disorders, Iran, Systematic Review, Meta-Analysis
Volume 6 Issue 4 - 2018

\author{
Vahideh Takhviji,' Ebrahim Azizi, ${ }^{2}$ Ali \\ Kordian, ${ }^{2}$ Abbas Khosravi, ${ }^{2}$ Mostafa Paridar, ${ }^{3}$ \\ Mohammad Ali Jalalifar, ${ }^{2}$ Omid Kiani \\ Ghalehsardi, ${ }^{4}$ Mohammad Abdollahi, ${ }^{5}$ Mofid \\ Hosseinzadeh, ${ }^{6}$ Zahra Bakshandeh ${ }^{5}$ \\ 'Laboratory Hematology and Blood Banking, Shahid Beheshti \\ University of Medical Science, Iran \\ ${ }^{2}$ Research Center for Thalassemia and Hemoglobinopathy, Ahvaz \\ Jundishapur University of Medical Sciences, Iran \\ ${ }^{3}$ Deputy of Management and Resources Development, Iran \\ ${ }^{4}$ Department of Hematology, Iran University of Medical \\ Sciences, Iran \\ ${ }^{5}$ Transfusion Research center, Iran \\ ${ }^{6}$ Emergency Medicine Department, Ahvaz Jundishapur University \\ of Medical Sciences, Iran
}

\begin{abstract}
Correspondence: Zahra Bakshandeh, Transfusion Research center, High Institute for Research and Education in Transfusion Medicine, Tehran, Iran, Email z.bakhshandeh.2009@gmail.com
\end{abstract}

Received: July 02, 2018 | Published: August 31, 2018

\section{Introduction}

Among blood products transmitted viral infections, hepatitis $\mathrm{C}$ as the most important cause of post-transfusion complication corresponding to the $90 \%$ of post transfusion hepatitis. ${ }^{1}$ Patients who require multi blood transfusion like patients with hereditary bleeding disorders and major thalassemia are noticeably at high risk of transfusion-transmitted infections. ${ }^{2,3}$ Hepatitis $\mathrm{C}$ virus is a single-stranded RNA virus which is the main cause of non-A non-B hepatitis recognized in $1989 .{ }^{4} \mathrm{HCV}$ infection affected about $2-3 \%$ of the population and is a global health problem. ${ }^{5}$ Clinically, it presents in two acute and chronic forms. Acute form is usually asymptomatic and rarely accompanies life-threatening conditions. Also, fifteen to forty-five percent of cases are treated within 6 months without any treatment. On the other hand $60 \%$ to $80 \%$ of subjects develop chronic form of disease which is a leading cause of liver cirrhosis within 20 years. ${ }^{6} \mathrm{HCV}$ as the main cause of chronic liver disease is responsible for the most hepatocellular carcinomas and requires intensive care.
Hereditary bleeding diseases (i.e. Hemophilia, Von Willebrand disease, clotting factors deficiency and etc.) are a group of conditions that result when the coagulation system doesn't function properly and patients experience extended bleeding after injury or even spontaneously. Thalassemia is a group of inherited disorders of the globin chain synthesis in which hemoglobin is reduced or absent. Thalassemia is classified based on the type of affected chain (alpha( $\alpha)$ or $\operatorname{beta}(\beta)$ thalassemia $\&$...). Major $\beta$-thalassemia reveals with a severe anemia about 6 months after birth and in the absence of treatment causes developmental disorder and skeletal malformation and ultimately reduces the patient's life. Therefore, regular blood transfusions are deemed necessary in these patients. ${ }^{7}$

Prior to 1990, almost all patients with hereditary coagulation disorders received HCV infected pooled plasma products. ${ }^{2}$ Since 1990, screening tests have been available for Anti-HCV screening to reduce probability of transmission of this virus through the blood transfusions, but screening tests aren't able to detect virus during the window 
period. ${ }^{8}$ The transmission of viral infection by blood products depends on a variety of factors, including the prevalence of infection in the population, the prevalence of infection in donors, screening tests and vaccination status. Although vaccination against $\mathrm{HBV}$ and screening tests have played a greater role in controlling the HBV transmission, but HCV has already remained as a massive challenge and a major cause of post transfusion hepatitis in multi-transfused patients. This systematic review and meta-analysis study aimed to determine hepatitis $\mathrm{C}$ virus prevalence among patients with thalassemia and inherited bleeding disorders in Iran.

\section{Materials and methods}

\section{Search strategy}

The current meta-analysis study achieved by the "Preferred Reporting Items of Systematic Reviews and Meta-Analysis" PRISMA guidelines. ${ }^{9}$ Following, biological and health sciences databases were searched: Pubmed, Scopus, EMBASE, Ebsco, Science Citation Index Expanded, Ovid, Google Scholar, Iran Medex, Magiran And Scientific Information Database (SID). The keywords were applied for search: "Viral Infection" OR "Hepatitis C" OR "HCV" AND "Prevalence" OR "Frequency" OR "Epidemiology" AND "Thalassemia" OR "Hemophilia" OR "Inherited Bleeding Disorders" OR "Multi-Transfused" OR "Blood Transfusion" AND "Iran". Moreover, reference lists of detected articles, reviews and meta-analysis were investigated for further eligible studies.

\section{Inclusion and exclusion criteria}

First, the titles and abstracts of the studies reviewed by tow trained researchers independently. The inclusion criteria were:

i. Studies surveying the HCV prevalence among Iranian provinces.

ii. Studies evaluated just Patients who receive blood products frequently.

iii. Studies used only validated diagnostic tests, including Polymerase Chain Reaction (PCR) and Recombinant Immunoblot Assay (RIBA) with the same Sensitivity and specificity.

iv. Studies written in English and Persian.

v. Observational studies like cross-sectional, case-control, prospective and retrospective.

The exclusion criteria were as follows: i) studies non-carried out among the Iranian population, ii) studies non-included enough data to assess the HCV prevalence, iii) studies used unvalidated diagnostic tests, including serological assays or Enzyme Linked Immunosorbent Assay (ELISA), iv) studies evaluating other risk factors of HCV transmission, v) identical and unrelated studies and vi) case-series, case reports, reviews, books, conference abstracts, editorial commentary. Afterward, the full text of the elected studies reviewed and observed contradictions resolved through discussion. Our research was performed from database inception up to January 2017.

\section{Data extraction and quality assessment}

The following retrieved study data were: title, first author name, year of publication, city of study, sample size and virus detection method. The quality of studies was evaluated using the Newcastle Ottawn Scale (NOS).$^{10}$ based on the scores, studies classified into three low, moderate and high-quality study with 0-3, 4-6 and 7-9 score, respectively.

\section{Statistical analysis}

We used Cochran Q and the I2 statistics to evaluate heterogeneity between the included studies $(\mathrm{I} 2=(\mathrm{Q}-\mathrm{df}) / \mathrm{Q} \times 100 \%$; I $2<25 \%$, no heterogeneity; $\mathrm{I} 2=25-50 \%$, moderate heterogeneity; $\mathrm{I} 2=50-75 \%$, large heterogeneity, I $2>75 \%$, extreme heterogeneity). Accordingly, heterogeneity was considered significant if the Q statistic had $\mathrm{p}<0.1$ or I $2>50 \%$. In the presence of significant heterogeneity, the random-effects model was applied; otherwise, the fixed-effect model was performed to combine the data.

The meta-analysis was performed using software version STATA 14.1. HCV prevalence with $95 \%$ confidence interval (CI) calculated by the Der Simonia-Laired random effects model. ${ }^{11} \mathrm{I}^{2}$ test used to assess the heterogeneity among included articles. ${ }^{12}$ A sensitivity analysis carried out to assess the stability of the results and Egger's regression test used to evaluate publication bias. A P-value less than 0.05 considered statically significant.

\section{Results}

Forty-seven studies, composed of 12449 patients; 8673 thalassemia and 3776 patients with inherited bleeding disorders (mainly hemophilia), fulfilled our criteria and included in the meta-analysis. The year of data collection in the studies were between 1998 to 2015 (Table 1).

The pooled HCV prevalence was estimated $28 \%$ (95\% confidence intervals $[\mathrm{CI}]=22 \%-33 \%$ ). The prevalence in patients with thalassemia and inherited bleeding disorders were $19 \%$ ([CI] $95 \%=16 \%$ $23 \%$ ) and $42 \%([\mathrm{CI}] 95 \%=33 \%-52 \%)$, respectively (Figure 1$)$. There was a considerable heterogeneity between studies. The highest prevalence of $\mathrm{HCV}$ infection (54\%) in patients with bleeding disorders was observed in the Tehran province and the lowest rate $(6 \%)$ in West Azerbaijan province (Figure 2). The prevalence trend of $\mathrm{HCV}$ in the patients assessed from 2000 to 2012 years. The findings of this assessment showed that the prevalence varies from year to year, but the trend was not logical (Figure 3).

Table I Summary of included studies

\begin{tabular}{llllllll}
\hline Author & Year & Region & SS & Disease & HCVpos & Method & Quality of study \\
\hline Samimi-Rad & 2006 & Yazd & 77 & bleeding disorders & 41 & RIBA & high \\
Samimi-Rad & 2006 & Yazd & 93 & Thalassemia & II & RIBA & high \\
Tamaddoni, & 2006 & Babol & 113 & Thalassemia & 12 & ELISA & Medium \\
Kalantari & 2010 & Isfahan & 545 & Thalassemia & 31 & RNA & high \\
Kalantari & 2010 & Isfahan & 615 & bleeding disorders & 347 & RNA & high \\
\hline
\end{tabular}


Table Continued..

\begin{tabular}{|c|c|c|c|c|c|c|c|}
\hline Author & Year & Region & SS & Disease & HCVpos & Method & Quality of study \\
\hline Shari -Mood, & 2006 & Zahedan & 81 & bleeding disorders & 24 & RIBA & High \\
\hline Jafroodi & 2012 & Guilan & 1113 & Thalassemia & $|5|$ & RIBA & High \\
\hline Assarehzadegan & 2009 & Khuzestan & 87 & bleeding disorders & 42 & RNA & High \\
\hline Valizadeh & 2010 & West Azarbaijan & 50 & bleeding disorders & 3 & RIBA & High \\
\hline Ansar & 1998 & Rasht & 67 & Thalassemia & 50 & RIBA & High \\
\hline Hassanshahi & 2011 & Southeastern & 181 & Thalassemia & 81 & RNA & High \\
\hline Azarkeivan & 2011 & Tehran & 395 & Thalassemia & 109 & RIBA & High \\
\hline Yazdani & 2010 & Isfahan & 350 & bleeding disorders & 232 & RIBA & High \\
\hline Sanei moghdam & 2003 & Zahedan & 364 & Thalassemia & 49 & RIBA & High \\
\hline Torabi & 2003 & East Azarbaijan & 84 & Thalassemia & 6 & RIBA & High \\
\hline Shahshahani & 2003 & Yazd & 85 & Thalassemia & 8 & RIBA & High \\
\hline Shahshahani & 2003 & Yazd & 74 & bleeding disorders & 36 & RIBA & High \\
\hline Azarkeivan & 2010 & Tehran & 395 & Thalassemia & 109 & RIBA & High \\
\hline Pedram & 2006 & Ahvaz & 634 & Thalassemia & & & \\
\hline Kasraian & 2011 & Isfahan & 570 & Thalassemia & 60 & RIBA & High \\
\hline Kasraian & 2011 & Isfahan & 350 & bleeding disorders & 232 & RIBA & High \\
\hline Karimi & 2001 & Shiraz & 466 & Thalassemia & 73 & RIBA & High \\
\hline Karimi & 2001 & Shiraz & 755 & Thalassemia & & & \\
\hline Mirmomen & 2006 & Multicenteric & 732 & Thalassemia & $14 \mid$ & RIBA & High \\
\hline Sayad & 2015 & Kermanshah & 232 & Thalassemia & 14 & RIBA & High \\
\hline Kazemi Arababadi & 2008 & Kerman & 60 & Thalassemia & 27 & RNA & high \\
\hline Alavian & 2003 & Qazvin & 103 & Thalassemia & 23 & RIBA & High \\
\hline Mahdaviani & 2004 & Markazi & 97 & Thalassemia & 7 & RIBA & High \\
\hline Mahdaviani & 2004 & Markazi & 68 & bleeding disorders & 25 & RIBA & High \\
\hline Akbari & 2004 & Shiraz & 200 & Thalassemia & 50 & RIBA & High \\
\hline Alavi & 2002 & Tehran & 110 & Thalassemia & II & RNA & High \\
\hline Alizadeh & 2006 & Hamedan & 66 & bleeding disorders & 28 & RNA & High \\
\hline Boroujerdnia & 2007 & Khuzestan & 206 & Thalassemia & 46 & RNA & High \\
\hline Bozorg & 2005 & Qazvin & 207 & Thalassemia & 50 & RIBA & High \\
\hline Esfahani & 2012 & Hamedan & 77 & bleeding disorders & 15 & RNA & High \\
\hline Faranoush & 2002 & $\begin{array}{l}\text { Semnan, Damghan, } \\
\text { Garmsar }\end{array}$ & 63 & Thalassemia & 12 & RNA & High \\
\hline Keshvari & 2014 & Tehran & 257 & Thalassemia & 103 & ELISA & Medium \\
\hline Kashanchi Langarodi & 2010 & Karaj & 206 & Thalassemia & 29 & RIBA & High \\
\hline Mobini & 2006 & Yazd & 77 & bleeding disorders & 38 & RIBA & High \\
\hline Mousavian & 2006 & Tehran & 1095 & bleeding disorders & 593 & RNA & High \\
\hline Maziar Mojtabavi & 2006 & Isfahan & 553 & bleeding disorders & 125 & RNA & High \\
\hline Najafi & 1998 & Ghaem Shahr & 100 & Thalassemia & 18 & ELISA & Medium \\
\hline Sammak & 2007 & Qom & 142 & Thalassemia & 19 & RIBA & High \\
\hline Samimi-Rad & 2007 & Markazi & 98 & Thalassemia & 5 & RNA & High \\
\hline Samimi-Rad & 2007 & Markazi & 76 & bleeding disorders & 33 & RNA & High \\
\hline Ziaee & 2000 & Khorasan & 80 & bleeding disorders & 25 & RNA & High \\
\hline
\end{tabular}




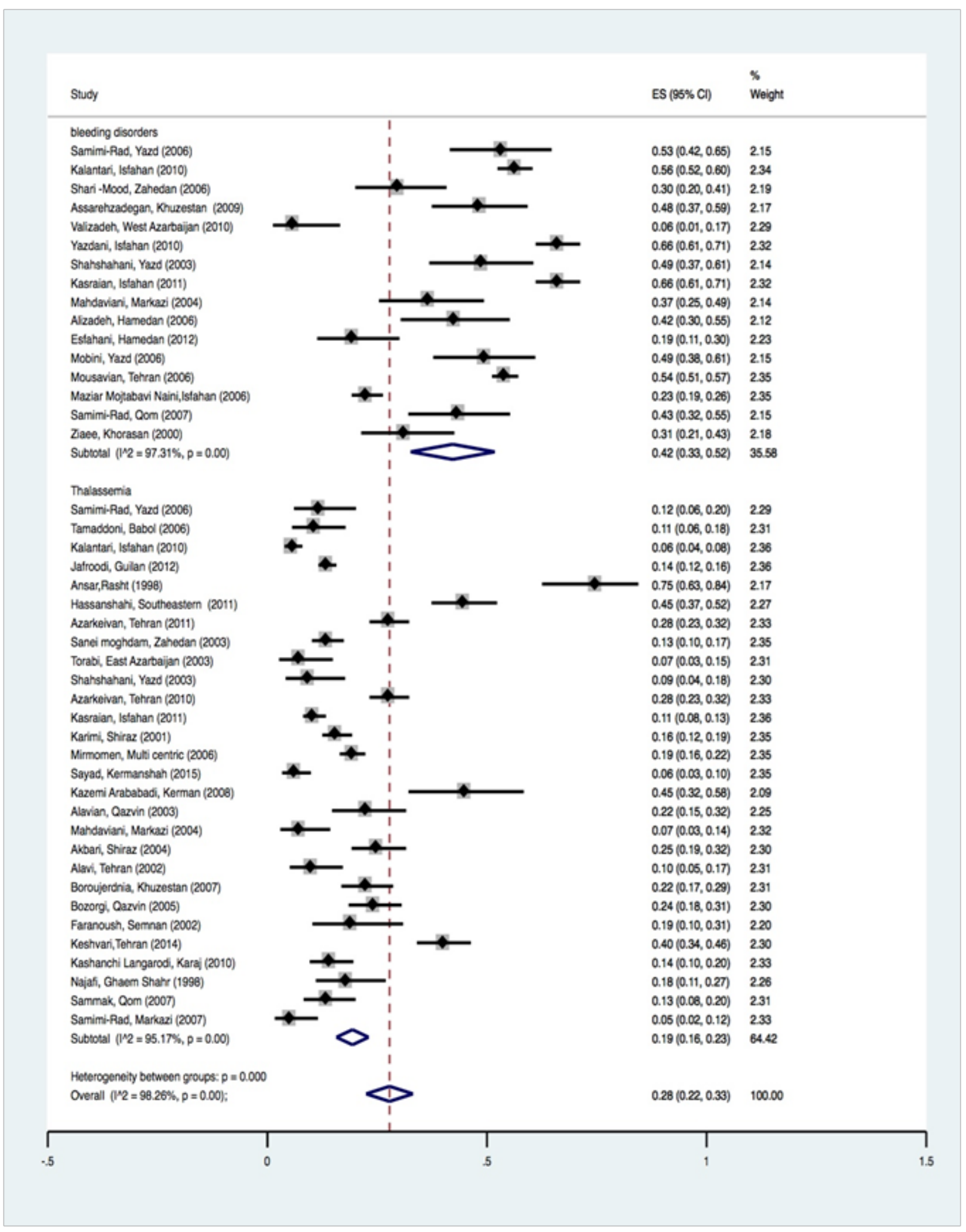

Figure I Prevalence of $\mathrm{HCV}$ in multi transfused patients.

Citation: Takhviji V, Azizi E, Kordian A, et al. Hepatitis C virus prevalence among patients with thalassemia and inherited bleeding disorders in Iran: a systematic review and meta-analysis. Hematol Transfus Int J. 2018;6(4):163-169. DOI: I0.15406/htij.2018.06.00I75 


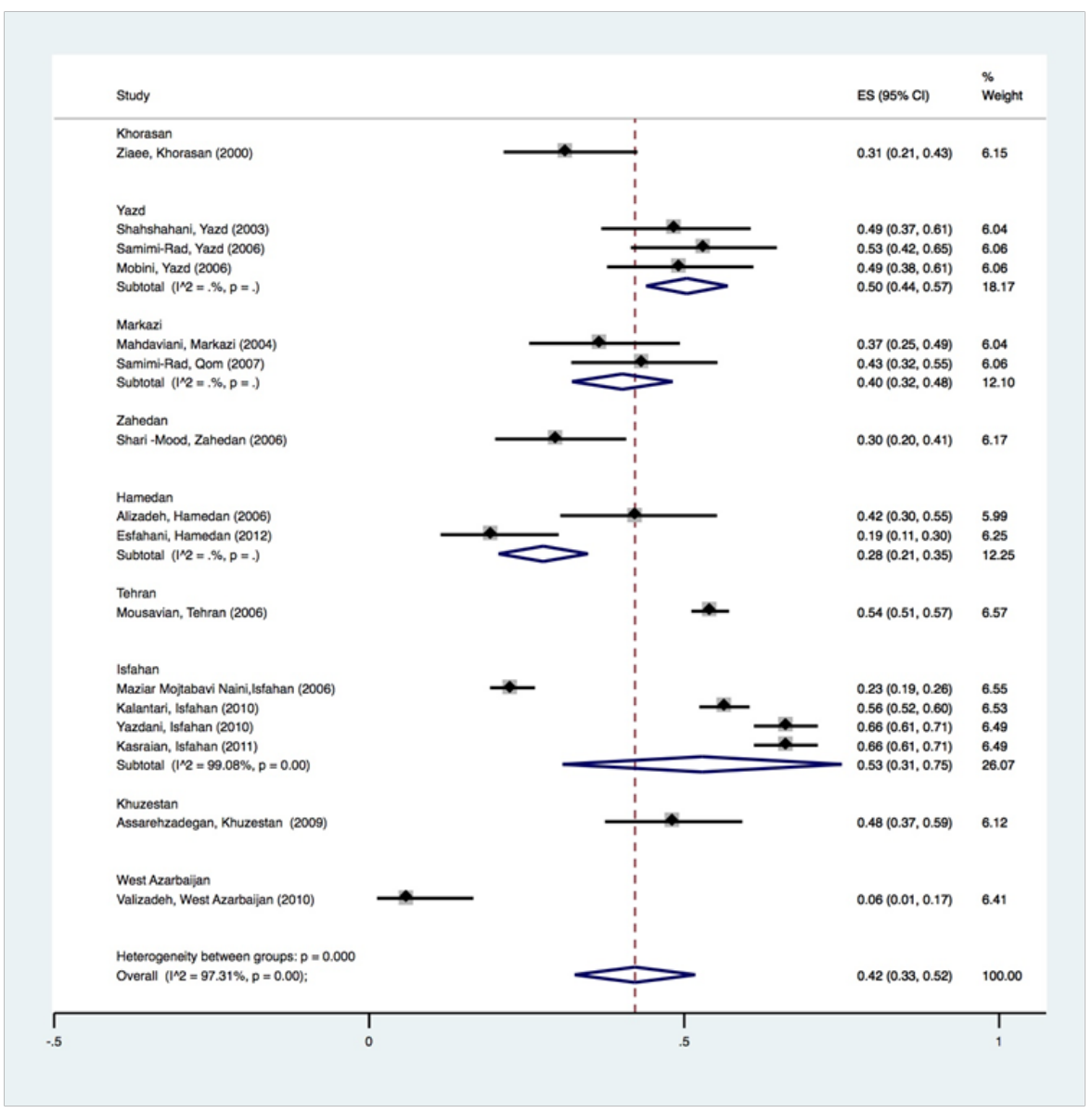

Figure 2 Prevalence of $\mathrm{HCV}$ in patients with bleeding disorders subgrouping by region.

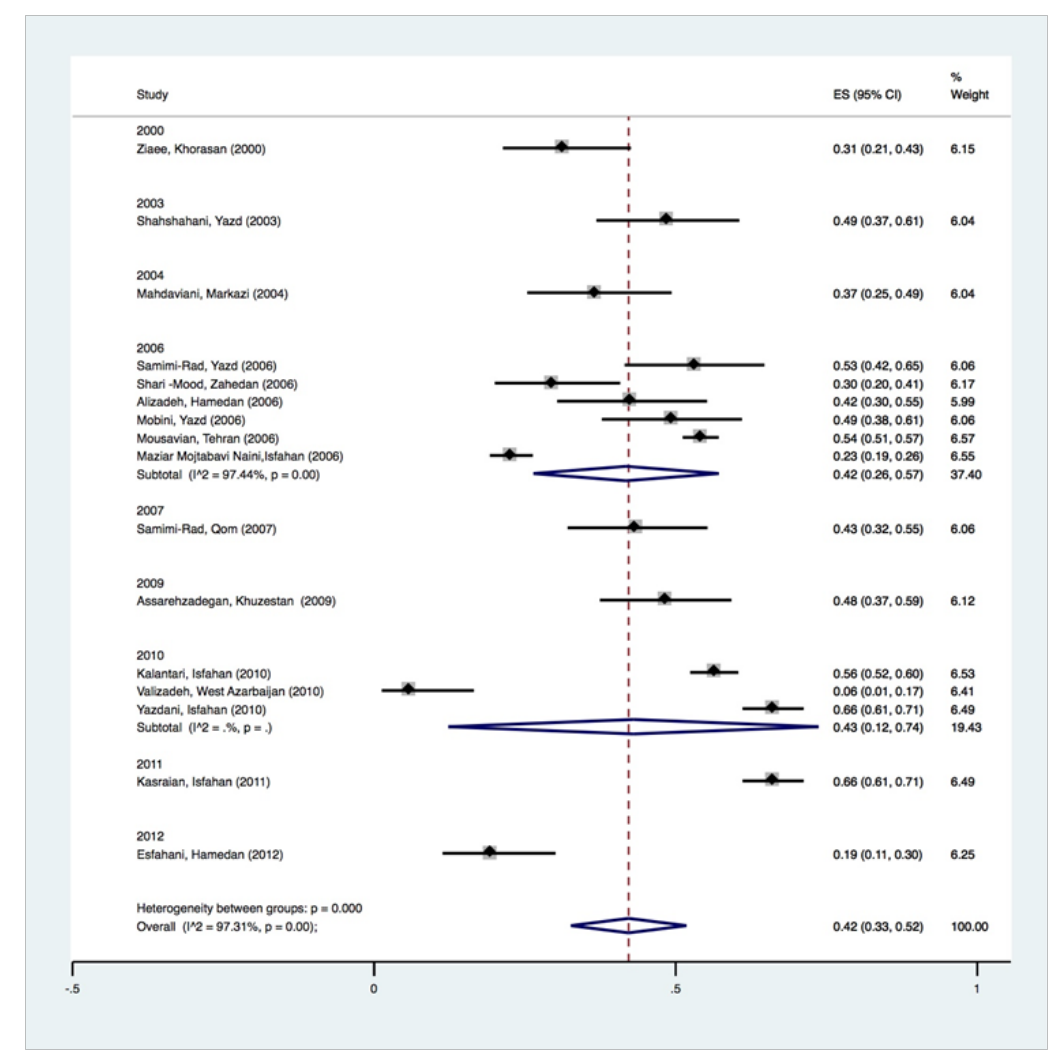

Figure 3 Prevalence of $\mathrm{HCV}$ in patients with bleeding disorders subgrouping by study year.

Citation: Takhviji V, Azizi E, Kordian A, et al. Hepatitis C virus prevalence among patients with thalassemia and inherited bleeding disorders in Iran: a systematic review and meta-analysis. Hematol Transfus Int J. 2018;6(4):163-169. DOI: I0.15406/htij.2018.06.00I75 
blood transfusion is an important risk factor for $\mathrm{HCV}$ infection among the multi-transfusion patients, accurate screening of donors is very effective to prevent the transmission of viral infections.

After using the 3rd Generation ELISA kits in IBTO (Iranian Blood Transfusion Organization) for detection of anti-HCV, the prevalence of HCV among the multi-transfusion patients reduced significantly. In a study conducted by Mirmomen and colleagues (2006), the prevalence of $\mathrm{HCV}$ infection in $\beta$-thalassemia patients ranged from $22.8 \%$ before the screening, to $2.6 \%$ after the screening of donated bloods. ${ }^{16}$ Given that multi-transfusion patients are exposed to blood-borne infections during their life, using advanced screening techniques on donated blood plays an important role in preventing the transmission of infection. Also, regular monitoring of the patients would be helped with early detection of the transmitted infections in these patients.

\section{Acknowledgements}

The authors would like to thank all of the research staff at Thalassemia and Hemoglobinopathy Research Center of Ahvaz University of Medical Sciences who helped to recruit patients and perform the experiments.

\section{Conflict of interest}

The authors declare that they have no conflict of interest.

\section{References}

1. Namasopo SO, Ndugwa C, Tumwine JK. Hepatitis C and blood transfusion among children attending the Sickle Cell Clinic at Mulago Hospital, Uganda. Afr Health Sci. 2013;13(2):255-260.

2. Papadopoulos N, Argiana V, Deutsch M. Hepatitis C infection in patients with hereditary bleeding disorders: epidemiology, natural history, and management. Ann Gastroenterol. 2018;31(1):35-41.

3. Sayad B, Foroughikia M, Akramipour R, et al. Hepatitis B, Hepatitis $\mathrm{C}$, and Human Immunodeficiency Virus Infection in Multi-Transfused Thalassemic Patients, Kermanshah, Iran, 2015. Jundishapur J Microbiol. 2017;10(5):e46152.

4. Petruzziello A, Marigliano S, Loquercio G, et al. Global epidemiology of hepatitis $\mathrm{C}$ virus infection: An up-date of the distribution and circulation of hepatitis C virus genotypes. World $J$ Gastroenterol. 2016;22(34):7824-7840.

5. Benova L, Mohamoud YA, Calvert C, et al. Vertical transmission of hepatitis $\mathrm{C}$ virus: systematic review and meta-analysis. Clin Infect Dis. 2014;59(6):765-773.

6. Tong TN, Branch DR. Use of a Monocyte Monolayer Assay to Evaluate Fc $\gamma$ Receptor-mediated Phagocytosis. J Vis Exp. 2017;(119).

7. Fibach E, Rachmilewitz EA. Pathophysiology and treatment of patients with beta-thalassemia-an update. F1000Res. 2017;6:2156.

8. Koerner K, Cardoso M, Dengler T, et al. Estimated risk of transmission of hepatitis C virus by blood transfusion. Vox Sang. 1998;74(4):213-216.

9. Moher D, Liberati A, Tetzlaff J, et al. Preferred reporting items for systematic reviews and meta-analyses: the PRISMA statement. Ann Intern Med. 2009;151(4):264-269,

10. Stang A. Critical evaluation of the Newcastle-Ottawa scale for the assessment of the quality of nonrandomized studies in meta-analyses. Eur $J$ Epidemiol. 2010;25(9):603-605.

11. Sadeghi F, Salehi-Vaziri M, Almasi-Hashiani A, et al. Prevalence of hepatitis $\mathrm{C}$ virus genotypes among patients in countries of the eastern Mediterranean regional office of WHO (EMRO): a systematic review and meta-analysis. Hepat Mon. 2016;16(4):e35558. 
12. Abolghasemi H, Amid A, Zeinali S, et al. Thalassemia in Iran: epidemiology, prevention, and management. J Pediatr Hematol Oncol. 2007;29(4):233-238.

13. Samimi-Rad K, Rahimnia R, Sadeghi M, et al. Epidemic History of Hepatitis C Virus among Patients with Inherited Bleeding Disorders in Iran. PLoS One. 2016;11(9):e0162492.

14. Khan MM, Tait RC, Kerr R, et al. Hepatitis C infection and outcomes in the Scottish haemophilia population. Haemophilia. 2013;19(6):870-875.

15. Seyed-Moayed Alavian. Hepatitis C infection in Iran; A review article. Iranian Journal of Clinical Infectious Diseases. 2009;4(1):47-59.

16. Mirmomen S, Alavian SM, Hajarizadeh B, et al. Epidemiology of hepatitis $\mathrm{B}$, hepatitis $\mathrm{C}$, and human immunodeficiency virus infecions in patients with beta-thalassemia in Iran: a multicenter study. Arch Iran Med. 2006;9(4):319-323.

17. Alavian SM, Tabatabaei SV, Lankarani KB. Epidemiology of HCV Infection among Thalassemia Patients in Eastern Mediterranean Countries: a Quantitative Review of Literature. Iran Red Crescent Med J. 2010;12(4):365-376.
18. Jafroodi M, Davoudi-Kiakalayeh A, Mohtasham-Amiri Z, et al. Trend in prevalence of hepatitis $C$ virus infection among $\beta$-thalassemia major patients: 10 years of experience in Iran. Int J Prev Med. 2015;6:89.

19. Kadhim KA, Baldawi KH, Lami FH. Prevalence, Incidence, Trend, and Complications of Thalassemia in Iraq. Hemoglobin. 2017;41(3):164-168.

20. Al-Naamani K, Al-Zakwani I, Al-Sinani S, et al. Prevalence of hepatitis $\mathrm{C}$ among multi-transfused thalassaemic patients in Oman: Single centre experience. Sultan Qaboos Univ Med J. 2015;15(1):e46-e51.

21. Atwa ZT, Abdel Wahed WY. Transfusion transmitted infections in frequently transfused thalassemic children living in Fayoum Governorate, Egypt: Current prevalence and risk factors. J Infect Public Health. 2017;10(6):870-874.

22. Chainuvati T, Poovorawan Y, Luengrojanakul P. The prevalence of hepatitis $\mathrm{C}$ virus antibody in high risk group of Thai children and adults. Gastroenterologia Japonica. 1991;26(Suppl 3):176-178. 